\title{
2021 MRS Fall Meeting \& Exhibit designed as a hybrid event
}

\author{
mrs.org/fall2021
}

B uilding on the success of the two recent Materials Research Society (MRS) Virtual Meetings, the Society takes the 2021 MRS Fall Meeting \& Exhibit to a new level, offering both in-person (Nov. 29-Dec. 2) and virtual (Dec. 6-8) meeting components.

The on-site experience in Boston, Mass. will include in-person opportunities to hear leading-edge research, meet with colleagues, and explore a robust Exhibit Hall. Select content from the in-person meeting will be live-streamed to the virtual audience on November 29-December 2, and in-person meeting attendees will also enjoy access to the full virtual experience on December 6-8. The virtual experience will bring exceptional meeting content to attendees worldwide with live online, prerecorded, and on-demand scientific sessions.

The 2021 MRS Fall Meeting Chairs are Markus J. Buehler, Massachusetts Institute of Technology; Craig Fennie, Cornell University; Marina S. Leite, University of California, Davis; Laura Na Liu, Universität Stuttgart; and Cengiz S. Ozkan, University of California, Riverside.

\section{Technical sessions}

The 59 symposia are grouped into seven topical clusters. The cluster on Broader Impact features two symposia. The first will focus on developing an open source introductory materials science textbook. The second will highlight and celebrate women in materials science and their impact on and contributions to the field - this will feature a number of invited talks by pioneers, leaders, and up-and-coming female researchers.

The Characterization cluster includes symposia on in situ and operando techniques applied to electrochemical systems; solid-state chemistry of new materials; scanning probe microscopy techniques; and the use of machine learning methods to enhance and accelerate the characterization of materials. Machine learning approaches for materials development will be further expanded in the cluster on Materials Computing and Data Science.

The largest cluster of the MeetingElectronics, Optics and Quantumincludes 20 symposia covering electronic materials/devices, optical materials, and quantum materials and processes, with several symposia cutting across these areas, such as quantum optical materials based on impurities. The 14 symposia in the cluster on Energy and Sustainability feature the applications of materials to energy transfer, conversion and storage, as well as addressing issues of materials design and characterization. The theme of sustainability cuts across the symposia in this cluster. Another large cluster is Biomaterials and Soft Materials that offers 12 symposia. One timely symposium focuses on antimicrobial materials against coronaviruses and other nosocomial pathogens.
With a total of four symposia in the cluster on Structural and Functional Materials, three of these focus on additive manufacturing, for emerging applications; functional materials and devices; and polymers, composites, and hybrids. The fourth symposium covers current trends in atomic layer deposition and chemical vapor deposition.

The long-running Symposium $\mathbf{X}$ Frontiers of Materials Research will feature presentations aimed at a broad audience and on topics at the forefront of research in materials science and engineering. To complement the symposia, Tutorial Sessions will provide detailed information on areas of research, and the Exhibit will showcase products and services of interest to the research community.

\section{Award recipients Von Hippel Award}

Harry Atwater (California Institute of Technology, USA) "for fundamental research in light-matter interactionsparticularly nanophotonics, plasmonics, photonic metamaterials, and solar energy conversion-and numerous applications of photon control of materials illustrating the value of fundamental research to technologies that improve the quality of life."

\section{David Turnbull Lectureship}

Nicholas A. Kotov (University of Michigan, USA) "for foundational discoveries in interface-based engineering of self-organizing materials." 


\section{MRS Medal}

Yury Gogotsi (Drexel University, USA) "for contributions to advancing the understanding of processing, structure, and properties of two-dimensional carbides and nitrides (MXenes) for energy storage applications."

\section{Materials Theory Award}

Emily Carter (Princeton University, USA) "for advances in quantum mechanics theory with broad applications to materials and chemical sciences."

\section{MRS Nelson "Buck" Robinson} Science and Technology Award for Renewable Energy

Stafford Sheehan (Air Company, USA) has been named to receive this award that recognizes a student, postdoc, or other early-career professional through five years following the highest degree attained for the development of novel sustainable solutions for the realization of renewable sources of energy.

\section{MRS Postdoctoral Awards}

Zhijie Chen (Northwestern University, USA) "for his outstanding contributions to the fields of porous materials, nanochemistry, and supramolecular assembly" and Dasha Nelidova (Institute of Molecular and Clinical Ophthalmology Basel, Switzerland) "for creating tunable nanogenetic near-infrared light sensors to restore vision."

\section{MRS Bulletin Postdoctoral Publication Prize}

Tedrick Thomas Salim Lew (Institute of Materials Research and Engineering [IMRE], Singapore) "for his excellent academic achievements, passion for his chosen area of research, interest in communicating his research and science to nonscientists, and significant leadership potential in the field of multidisciplinary materials science."
Kavli Awards

Sir J. Fraser Stoddart (Northwestern University, USA) will present The Fred Kavli Distinguished Lectureship in Materials Science during the Plenary session. Susan Bernal Lopez (University of Leeds, United Kingdom) is named to receive the The Kavli Foundation Early Career Lectureship in Materials Science.

\section{Distinguished Keynote}

Sir Kostya Novoselov (University of Manchester), a 2010 Nobel Laureate in Physics, will present the Distinguished Keynote address on the last day of The Virtual Experience, Dec. 8, at 9:00 am (EST).

\section{Stay up-to-date!}

To keep up to date on the symposium sessions, special events, registration, and visa and travel resources, visit mrs. org/fall2021. The website includes links for up-to-date information on traveling during COVID-19.

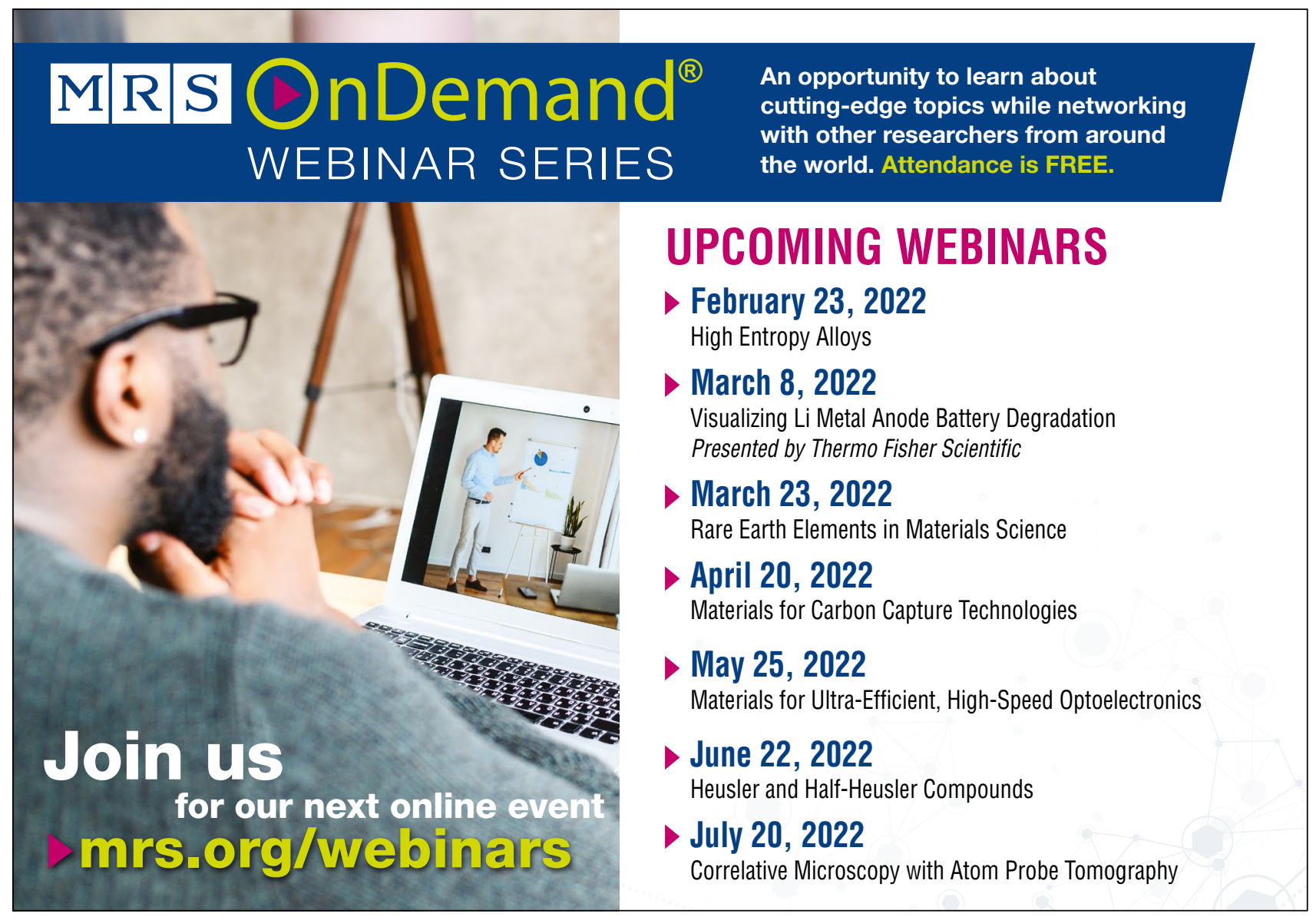

